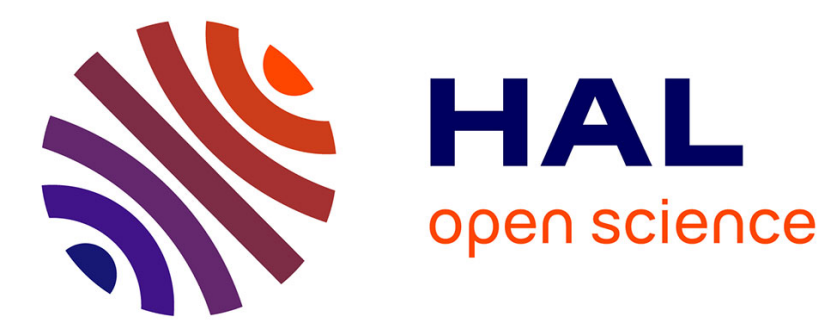

\title{
Charged particle acceleration by intermittent electromagnetic turbulence
}

L.M. Zelenyi, S.D. Rybalko, A.V. Artemyev, A.A. Petrukovich, G. Zimbardo

\section{To cite this version:}

L.M. Zelenyi, S.D. Rybalko, A.V. Artemyev, A.A. Petrukovich, G. Zimbardo. Charged particle acceleration by intermittent electromagnetic turbulence. Geophysical Research Letters, 2011, 38 (17), pp.L17110. 10.1029/2011GL048983 . hal-00655044

\section{HAL Id: hal-00655044 https://hal.science/hal-00655044}

Submitted on 26 Apr 2021

HAL is a multi-disciplinary open access archive for the deposit and dissemination of scientific research documents, whether they are published or not. The documents may come from teaching and research institutions in France or abroad, or from public or private research centers.
L'archive ouverte pluridisciplinaire HAL, est destinée au dépôt et à la diffusion de documents scientifiques de niveau recherche, publiés ou non, émanant des établissements d'enseignement et de recherche français ou étrangers, des laboratoires publics ou privés. 


\title{
Charged particle acceleration by intermittent electromagnetic turbulence
}

\author{
L. M. Zelenyi, ${ }^{1}$ S. D. Rybalko, ${ }^{2}$ A. V. Artemyev, ${ }^{1}$ A. A. Petrukovich, ${ }^{1}$ and G. Zimbardo ${ }^{3}$
}

[1] We studied the role of intermittency in the process of acceleration and transport of charged particles by electromagnetic turbulence. We propose a simple model of electromagnetic turbulence with a variable level of intermittency. The magnetic field is described as a superposition of an ensemble of magnetostatic plane waves and of spatially localized dynamic magnetic clouds. The amplitudes of magnetic clouds are distributed according to an intermittent map. The model approximates essential properties of turbulence observed 'in situ' in the neutral plane of the Earth's magnetotail. Numerical integration of charged particle trajectories in such a dynamic electromagnetic environment shows that, for the fixed time interval, the higher the level of intermittency, the higher the energy gain. Moreover, in a sufficiently intermittent turbulence, particle acceleration occurs without significant intensification of the spatial transport. Citation: Zelenyi, L. M., S. D. Rybalko, A. V. Artemyev, A. A. Petrukovich, and G. Zimbardo (2011), Charged particle acceleration by intermittent electromagnetic turbulence, Geophys. Res. Lett., 38, L17110, doi:10.1029/2011GL048983.

\section{Introduction}

[2] Numerous observations of high energy particles (up to several $100 \mathrm{keV}$ ) [Sarafopoulos et al., 2001; Haaland et al., 2010] point to the presence of various mechanisms of charged particles heating and acceleration inside the magnetotail. These mechanisms may be divided into two main groups: quasi-stationary processes (e.g., magnetic reconnection in the downtail region resulting in acceleration due to the cross-tail electrostatic field $E_{y}$ ) and various nonstationary processes, where, in fact, inductive electric fields are responsible for particle acceleration. One can mention stochastic heating by electromagnetic turbulence (EMT) in the current sheet [Perri et al., 2009; Artemyev et al., 2009], energization during the magnetic field dipolarization [Delcourt, 2002; Ono et al., 2009] and the acceleration due to the non-stationary magnetic reconnection [e.g., Hoshino, 2005; Retinò et al., 2008, and references therein]. Particles could gain only part of the dawn-dusk potential drop, which limits the efficiency of mechanisms from the first group to energies $<50-100 \mathrm{keV}$. Therefore, to describe acceleration of relatively small particle populations up to several hundred

\footnotetext{
${ }^{1}$ Space Research Institute, Russian Academy of Sciences, Moscow, Russia.

${ }^{2}$ FEMTO, Optics Department, UMR 6174, CNRS, Universite de Franche-Comte, Besancon, France.

${ }^{3}$ Dipartimento di Fisica, Universita della Calabria, Arcavacatadi Rende, Italy.
}

$\mathrm{keV}$, one needs to consider turbulent non-stationary mechanisms. On the one hand, regions in the magnetotail filled by turbulence are often strongly localized. On the other hand, turbulent acceleration is necessarily intrinsically coupled to the intensification of spatial transport, which again leads to a limitation in the maximum energy gain.

[3] Particle acceleration and transport in EMT are strongly dependent on the particular properties of the turbulence. In recent decades the main characteristics of EMT in the magnetotail have been obtained and published [Hoshino et al., 1994; Dudok de Wit and Krasnosel'skikh, 1996; Vörös et al., 2004; Petrukovich, 2005; Zimbardo et al., 2010]. It was shown that the observed EMT could not be described by the simple model of the Kolmogorov turbulence. EMT in space is intermittent and needs to be described by nonGaussian distribution functions of fluctuations. Therefore, the next logical step would be the investigation of the effect of these EMT properties on particle acceleration.

[4] The intermittency level for magnetic field $B(t)$ can be estimated with structure function analysis $S_{p}=\Sigma_{n} \mid B\left(t_{n}+\Delta\right)-$ $\left.B\left(t_{n}\right)\right|^{p}[$ Frisch, 1995; Dudok de Wit and Krasnosel'skikh, 1996]. This function can be approximated as $S_{p} \sim \Delta^{\zeta_{p}}$, where $\zeta_{p}$ is the power law exponent. For Kolmogorov turbulence we have $\zeta_{p} \sim p$ [Frisch, 1995]. If the $\zeta_{p}$ curve deviates from the linear behavior, then we are dealing with intermittent turbulence [e.g., Dudok de Wit and Krasnosel'skikh, 1996, and references therein].

[5] In this paper we propose the model of EMT with a variable level of intermittency (we define this level as the quantitative deviation of the power law exponent from the linear approximation $\zeta_{p} \sim p$ ). In the framework of this model we study efficiency of energization of charged particles and dependence of this energy gain on spatial transport.

\section{Model of EMT}

[6] We developed the EMT model with a simple 2D geometry of the neutral plane of the magnetotail current sheet (see discussion in the last section). Only one component of the magnetic field $B_{z}$ is present and we consider the motion of particles in the $(x, y)$ plane. The EMT model consists of two parts: the stationary turbulence with the power law spectrum $B_{(1)}(x, y)$ and the field generated by an ensemble of non-stationary localized magnetic structures (magnetic clouds) $B_{(2)}(x, y, t)$. The first part provides the power law spectrum of the turbulence conforming with the observations and corresponds to quasi-stationary magnetic fluctuations. The second part corresponds to the small-scale stochastic dynamical processes in the magnetotail and additionally supports the charged particle energization. We consider this representation of the turbulence as a practical way to take into account some typical effects. 


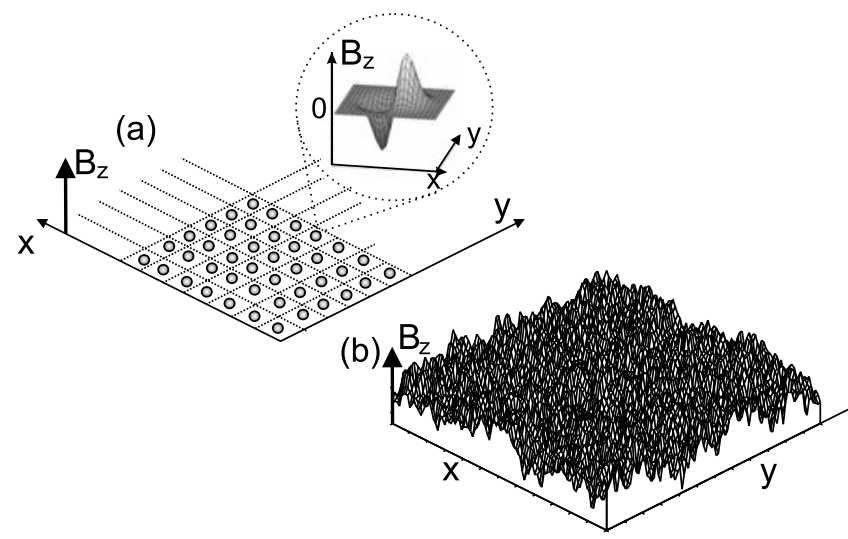

Figure 1. Scheme of the EMT model. Total field is a sum of (a) magnetic clouds component and (b) waves ensemble.

[7] To describe the first component of EMT we use the model of magnetostatic turbulence consisting of an ensemble of plane waves:

$$
B_{(1)}=B_{0} \sum_{s}\left(1+\left(\mathbf{k}_{\boldsymbol{s}} \mathbf{l}\right)^{2}\right)^{-\alpha} \cos \left(\mathbf{k}_{s} \mathbf{r}+\varphi_{0 s}\right)
$$

Here $\mathrm{B}_{0}$ is the amplitude of turbulence, $\mathbf{k}_{\mathrm{s}}=k_{s}\left(\cos \theta_{s} \mathbf{e}_{x}+\right.$ $\left.\sin \theta_{s} \mathbf{e}_{y}\right), \mathbf{r}=x \mathbf{e}_{x}+y \mathbf{e}_{y}, \mathbf{l}$ is the vector of the correlation scale [see Chiaravalloti et al., 2006], $\varphi_{0 s}$ are initial phases with random distribution in the range $[0,2 \pi]$. The magnitudes of the wavenumber $k_{s}$ are uniformly distributed in the range $[0.02,1] k_{\max }$ and each $k_{s}$ has twenty representatives propagating at different angles $\theta_{\mathrm{s}}$. The slope of the spectrum is $\alpha=5 / 8$ [Chiaravalloti et al., 2006]. We define the energy $W_{(1)}=\frac{1}{8 \pi} \int_{-L}^{L} \int_{-L}^{L} B_{(1)}^{2} d \mathbf{r}$, where $L=400 / k_{\max }$ is the maximal spatial scale imposed by the size of the modelling box. The snapshot of EMT defined by equation (1) is shown in Figure $1 b$.

[8] The second part of EMT is described by the model of magnetic clouds [Perri et al., 2007, 2009]. A single spatial localized burst of the magnetic field can be described by two components of the vector potential:

$$
A_{x, y}=a_{0} A_{(2)} \exp \left(-k_{c}^{2}\left(\mathbf{r}-\mathbf{r}_{0}(t)\right)^{2}\right)
$$

Here $a_{0}$ determines the absolute amplitude; $A_{(2)}$ is the amplitude of the vector potential for the given cloud; $1 / k_{c}=$ $0.5 / k_{\max }$ is the spatial scale of the given cloud; $\mathbf{r}_{0}(t)=\left(x_{0}+\right.$ $a \sin \omega t) \mathbf{e}_{x}+\left(y_{0}+a \sin \omega t\right) \mathbf{e}_{y}$, where $\left(x_{0}, y_{0}\right)$ is the initial coordinate of this cloud, $a=0.2 / k_{\max }$ and $\omega=0.25 q B_{0} / m c$ are the amplitude and the frequency of the cloud oscillations. The electromagnetic field is determined as $B_{(2)}=(\operatorname{curl} \mathbf{A}) \mathbf{e}_{z}$ and $\mathbf{E}=-c^{-1} \partial \mathbf{A} / \partial t$, where $\mathbf{A}$ is the total vector potential of all clouds.

[9] To distribute the magnetic clouds within our 2D modelling box, we divide the plane into the cells with the size $(\Delta l, \Delta l)$ (Figure 1a). At variance with the model of Perri et al. [2009], where the cloud positions are random, we place one cloud in each cell at $x_{0}=\Delta l\left(n_{x}+\frac{1}{2}\right), y_{0}=\Delta l\left(n_{y}+\frac{1}{2}\right)$ and $\Delta l k_{c}=8\left(1 / k_{c}=\Delta l / 8\right.$ and clouds do not overlap). Here $n_{x}=0 . . N_{\max }$ and $n_{y}=0 . . N_{\max }$ denote the cell number. In our simulation $N_{\max }=100$ and the total number of clouds is equal to $10^{4}$.
[10] The distribution of amplitudes $A_{(2)}$ over the spatial network $\left(n_{x}, n_{y}\right)$ determines the behavior of EMT. For example, the random distribution should give EMT with the Kolmogorov structure function. To obtain $\zeta_{p}$, which is typical for the intermittent turbulence, we design a special distribution for $A_{(2)}$. It is well known that a one-dimensional map $f_{n}=F\left(f_{n-1}\right)$ can exhibit the property of intermittency. Manneville [1980] showed that, depending on $\beta, F\left(f_{n-1}\right)=$ $f_{n-1}+f_{n-1}^{\beta}(\bmod 1)$ demonstrates the multitude of various dynamic regimes. When $1 \leq \beta<3 / 2$ the dynamics is normal, in the sense that the fluctuations of a random variable generated by the map are distributed according to Gaussian law. When $3 / 2<\beta<2$, the dynamics is transitional-anomalous, and when $\beta>2$ the dynamics is strictly anomalous, described by the Levy statistics with the index $1 /(\beta-1)$. The latter regime corresponds to the map with intermittency. Here we modify this map to obtain not only a positive but also a negative values of $f_{n}$ :

$$
f_{n+1}=F\left(f_{n}\right)=\operatorname{sgn}\left(f_{n}\right)\left[\left(\left|f_{n}\right|+\left|f_{n}\right|^{\beta}+1\right)(\bmod 2)-1\right]
$$

Here $f_{n} \in[-1,1], \beta \geq 1$. We used equation (3) to determine values of $A_{(2)}: A_{(2)}\left(n_{x}+1, n_{y}\right)=F\left(A_{(2)}\left(n_{x}, n_{y}\right)\right)+\hat{D}$ :

$$
\hat{D}=D\left(A_{(2)}\left(n_{x}, n_{y}-1\right)-2 A_{(2)}\left(n_{x}, n_{y}\right)+A_{(2)}\left(n_{x}, n_{y}+1\right)\right)
$$

where the diffusion coefficient $D$ provides the connection between values of $A_{(2)}$ at the net. The parameter $\beta$ allows to vary the level of the EMT intermittency. The parameter $a_{0}$ is defined in such a way that the energy $W_{(2)}=\frac{1}{8 \pi} \int_{-L}^{L} \int_{-L}^{L} B_{(2)}^{2} d \mathbf{r}$ is equal to $W_{(1)} / 5$ for all runs.

\section{Model Verification}

[11] To obtain the structure function for the EMT model, we use the following technique. At the randomly chosen time $t_{0}$ the magnetic field $B_{z}=B_{(1)}(x, y)+B_{(2)}\left(x, y, t_{0}\right)$ is calculated along the line $x=y$ with a spatial step $\Delta<1 / k_{\max }$. The series $B_{z}(n)\left(n\right.$ is the step number, $\left.n=1 . .5 \cdot 10^{6}\right)$ are taken to obtain the structure function $S_{p}=\Sigma_{n}\left|B_{z}(n+h)-B_{z}(n)\right|^{p}$. The approximation $S_{p} \sim h^{\zeta_{p}}$ gives the power law exponents $\zeta_{p}$ for various values of $\beta$ (Figure 2). The model parameter

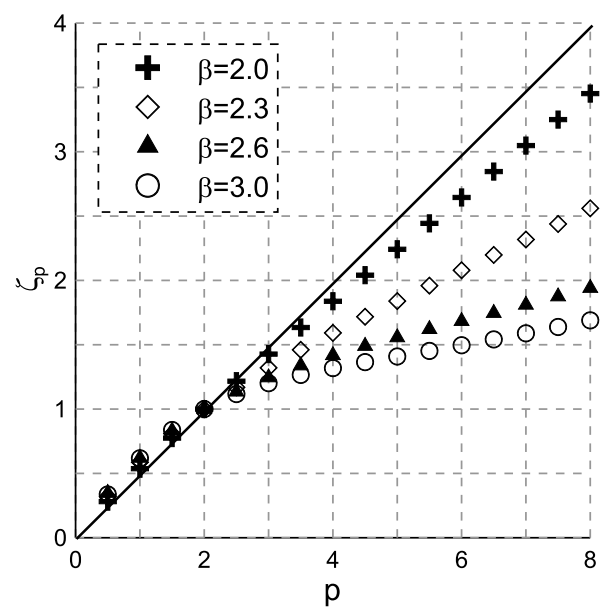

Figure 2. Power law exponent $\zeta_{p}$ as a function of its order $p$ for different values of $\beta$. 


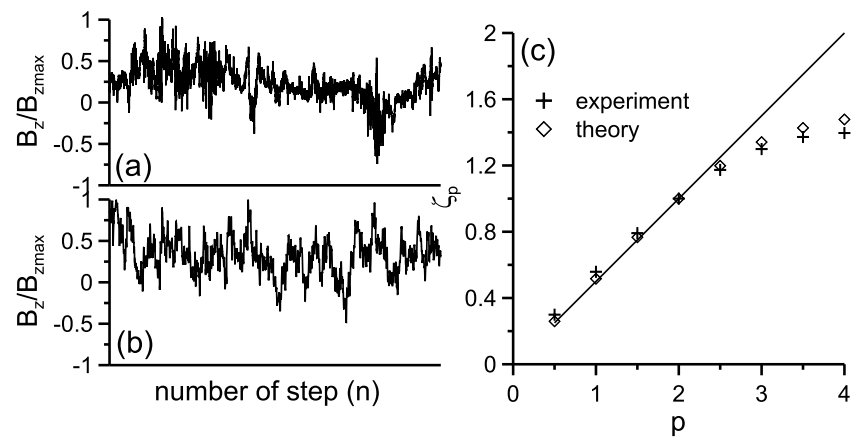

Figure 3. (a) The magnetic field observed by Interball [from Petrukovich, 2005, Figure 1.3]. (b) Model magnetic field. (c) Power law exponent of the model and observation.

$\beta$ controls the level of intermittency: an increase of $\beta$ led to the increase of the difference between $\zeta_{p}$ and the straight line $\zeta_{p} \sim p$.

[12] We compare this EMT model with the spacecraft observations obtained in the vicinity of the neutral plane of the Earth's magnetotail. The experimental values for $B_{z}$ and the corresponding $\zeta_{p}$ were taken from the paper by Petrukovich [2005]. The comparison between the observed and model magnetic fields are shown in Figures $3 \mathrm{a}$ and $3 \mathrm{~b}$. The related dependencies $\zeta_{p}$ are presented in Figure 3c. Although the model field does not contain the whole spectrum of the observed data (for the model we took into account only two orders of $k$ ), the theoretical profile $\zeta_{p}$ could be considered as a successful approximation. Therefore, the properties of the observed turbulence could be reasonably well reproduced by the developed model.

\section{Efficiency of Particle Acceleration}

[13] This section is devoted to the numerical simulations of particle acceleration and transport in EMT for various levels of intermittency. The trajectories of $10^{4}$ particles are integrated numerically. We use periodic boundary conditions. The time is normalized as $t \rightarrow \operatorname{tq} B_{0} / m c$, coordinates as $\mathbf{r} \rightarrow \mathbf{r} k_{\max }$ and energy $\varepsilon=\frac{1}{2}(d \mathbf{r} / d t)^{2}$ is normalized on $\left(q B_{0} / m c k_{\max }\right)^{2}$. We calculate an average energy of the particle ensemble $\langle\varepsilon(t)\rangle$ and average spatial displacement $R(t)=\left\langle\sqrt{(\mathbf{r}(t)-\mathbf{r}(0))^{2}}\right\rangle$ for various intermittency levels. Figure 4a demonstrates $\langle\varepsilon(t)\rangle$ : the increase of the intermittency level leads to the increase of energy gained by particles at a given time. Moreover, $\langle\varepsilon(t)\rangle$ as a function of $R(t)$ (Figure $4 \mathrm{~b}$ ) reveals a very important property of intermittent EMT: the energy gain by particles in the limited space region (for fixed $R(t)$ ) is larger for more intermittent turbulence. Therefore, the displacement $R(t)$ grows with an increase of the intermittency level slower than $\langle\varepsilon(t)\rangle$.

[14] We estimate the efficiency of particle acceleration which can be provided by the model under consideration for typical magnetotail parameters. The magnitude of magnetic field fluctuation $B_{0} \sim 10 \mathrm{nT}\left(q B_{0} / m c \sim 1 \mathrm{~s}^{-1}\right)$, the spatial scale of magnetic clouds $1 / k_{c} \sim 1000 \mathrm{~km}$ and $1 / k_{\max } \sim 500 \mathrm{~km}$, as a result $q B_{0} / m c k_{\max } \sim 500 \mathrm{~km} / \mathrm{s}$ and $\frac{1}{2}\left(q B_{0} / m c k_{\max }\right)^{2} \sim 1.5 \mathrm{keV}$. If we take $\beta \sim 3.0-3.5$, then particles gain $150 \mathrm{keV}(\varepsilon=100)$ within the region $R \sim(70-100) / k_{\max } \sim 6-8 R_{E}$. Increase of proton fluxes on the energy $\sim 100-500 \mathrm{keV}$ are often observed during active periods in the magnetotail [e.g., Ono et al., 2009; Haaland et al., 2010].

[15] Another interesting effect obtained here is the existence of two regimes of particle acceleration. The dependence of $\langle\varepsilon(t)\rangle$ on $t$ from Figure 4 demonstrates that $\langle\varepsilon(t)\rangle \sim t^{2}$ while $t<t^{*}$ and $\langle\varepsilon(t)\rangle \sim t$, if $t>t^{*}$ (for $\beta=3.5 t^{*} \approx 600$ ). The similar dependency of $\langle\varepsilon(t)\rangle$ was found by Zelenyi et al. [2008] in the non-intermittent EMT model. The first regime $\left(\langle\varepsilon(t)\rangle \sim t^{2}\right)$ can be described by the theory of resonant acceleration, when each particle interacts with a single cloud (or wave) for a sufficiently long time. When particle gains enough energy, it began to wander between different waves (clouds) and acceleration becomes diffusive with $\langle\varepsilon(t)\rangle \sim t$.

\section{Conclusions}

[16] In this paper we propose a 2D model of EMT with a controlled level of intermittency. This model allows us to estimate the effect of intermittency on particle transport and acceleration in the vicinity of the neutral plane of the Earth's magnetotail. Under the assumptions made, magnetic surfaces in the modelling box are strongly destroyed by the magnetic component of turbulence and charged particles can spend a sufficiently long time being trapped near the neutral plane. If the EMT is not strong enough to completely destroy the topology of field lines, particles should leave the neutral plane after a limited time interval [Speiser, 1967].

[17] The relationship between spatial transport and acceleration in intermittent EMT is one of the most interesting results obtained in this paper. We show that charged particles can gain more energy within the same spatial domain $R$ for more intermittent turbulence (for the same level of EMT energy). This result suggests a solution to the problem of

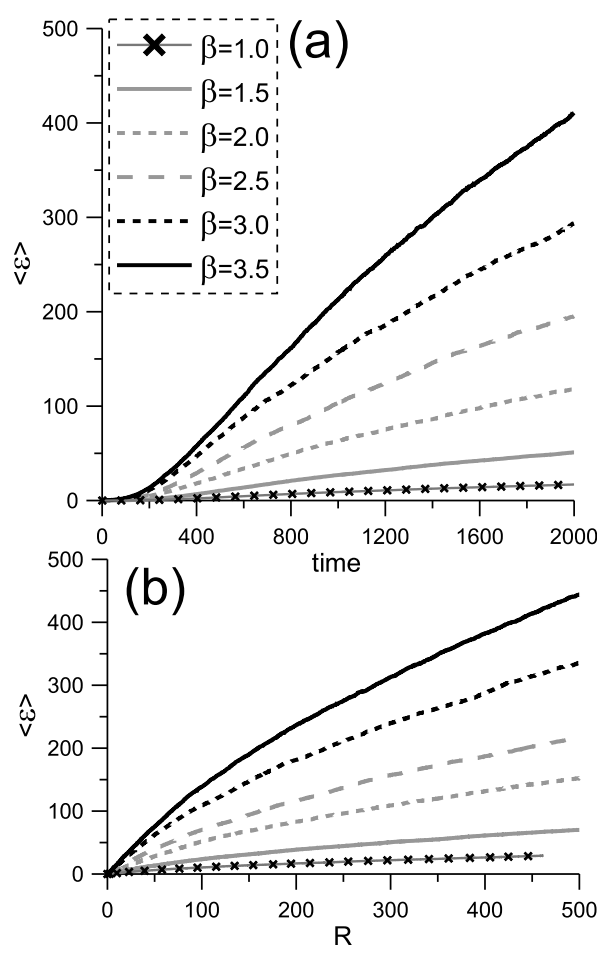

Figure 4. Average energy and displacement $R$ of particles ensemble as a function of (a) time and (b) displacement $R$ for various $\beta$. 
strong particle acceleration in spatially localized regions. Acceleration is necessarily associated with spatial transport for any of the EMT models [e.g., Perri et al., 2007; Zelenyi et al., 2008]. Thus, strong acceleration for non-intermittent turbulence is possible only if turbulence fills sufficiently large spatial regions. In contrast, intermittent EMT accelerates particles more effectively than it enhances spatial transport.

[18] The main results of this work are obtained by means of numerical simulation. Although this method allows the prompt estimate of the influence of intermittency, further investigations of intermittency effects on particle dynamics are needed. By developing an analytical theory, it should be possible to verify the results obtained. The main effects of non-intermittent EMT were already reproduced in analytical estimates [see Zelenyi and Milovanov, 2004, and references therein]. Now these theories should be generalized for the intermittent case.

[19] In conclusion, we developed a 2D model of EMT with a controlled level of intermittency which, based on the comparison of the model structure functions with actual data, is shown to approximate observations with reasonable accuracy. We show that the increase in the intermittency level leads to the increase in the acceleration efficiency in a limited time interval and in a limited spatial domain.

[20] Acknowledgments. Authors are grateful to Diane C. Taylor for her help in preparation of manuscript. The work was supported by the RF Presidential Program for State Support of Leading Scientific Schools (project NSh-3200.2010.2.), the Russian Foundation for Basic Research (projects 10-02-00135) by the European Union Seventh Framework Programme [FP7/2007-2013] under grant agreement 269198 Geoplasmas (Marie Curie International Research Staff Exchange Scheme).

[21] The Editor thanks Zoltan Voros and an anonymous reviewer for their assistance in evaluating this paper.

\section{References}

Artemyev, A.V., L. M. Zelenyi, H. V. Malova, G. Zimbardo, and D. Delcourt (2009), Acceleration and transport of ions in turbulent current sheets: Formation of non-Maxwellian energy distribution, Nonlinear Processes Geophys., 16, 631-639.

Chiaravalloti, F., A. V. Milovanov, and G. Zimbardo (2006), Self-similar transport processes in a two-dimensional realization of multiscale magnetic field turbulence, Phys. Scr. T, 122, 79-88.

Delcourt, D. C. (2002), Particle acceleration by inductive electric fields in the inner magnetosphere, J. Atmos. Sol. Terr. Phys., 64, 551-559.
Dudok de Wit, T., and V. V. Krasnosel'skikh (1996), Non-Gaussian statistics in space plasma turbulence: Fractal properties and pitfalls, Nonlinear Processes Geophys., 3, 262-273.

Frisch, U. (1995), Turbulence: The Legacy of A. N. Kolmogorov, Cambridge Univ. Press, Cambridge, U. K.

Haaland, S., et al. (2010), Spectral characteristics of protons in the Earth's plasmasheet: Statistical results from Cluster CIS and RAPID, Ann. Geophys., 28, 1483-1498.

Hoshino, M. (2005), Electron surfing acceleration in magnetic reconnection, J. Geophys. Res., 110, A10215, doi:10.1029/2005JA011229.

Hoshino, M., A. Nishida, T. Yamamoto, and S. Kokubun (1994), Turbulent magnetic field in the distant magnetotail: Bottom-up process of plasmoid formation?, Geophys. Res. Lett., 21, 2935-2938.

Manneville, P. (1980), Intermittency, self-similarity and $1 / \mathrm{f}$ spectrum in dissipative dynamical systems, J. Phys., 41, 1235-1243.

Ono, Y., M. Nosé, S. P. Christon, and A. T. Y. Lui (2009), The role of magnetic field fluctuations in nonadiabatic acceleration of ions during dipolarization, J. Geophys. Res., 114, A05209, doi:10.1029/ 2008JA013918.

Perri, S., F. Lepreti, V. Carbone, and A. Vulpiani (2007), Position and velocity space diffusion of test particles in stochastic electromagnetic fields, Europhys. Lett., 78, 40003, doi:10.1209/0295-5075/78/40003.

Perri, S., A. Greco, and G. Zimbardo (2009), Stochastic and direct acceleration mechanisms in the Earth's magnetotail, Geophys. Res. Lett., 36 , L04103, doi:10.1029/2008GL036619.

Petrukovich, A. A. (2005), Low frequency magnetic fluctuations in the Earth's plasma sheet, Astrophys. Space Sci. Libr., 321, 145-179.

Retinò, A., et al. (2008), Cluster observations of energetic electrons and electromagnetic fields within a reconnecting thin current sheet in the Earth's magnetotail, J. Geophys. Res., 113, A12215, doi:10.1029/ 2008JA013511.

Sarafopoulos, D. V., N. F. Sidiropoulos, E. T. Sarris, V. Lutsenko, and K. Kudela (2001), The dawn-dusk plasma sheet asymmetry of energetic particles: An Interball perspective, J. Geophys. Res., 106, 13,053-13,065.

Speiser, T. W. (1967), Particle trajectories in model current sheets: 2. Applications to auroras using a geomagnetic tail model, J. Geophys. Res., 72, 3919-3932.

Vörös, Z., et al. (2004), Magnetic turbulence in the plasma sheet, J. Geophys. Res., 109, A11215, doi:10.1029/2004JA010404.

Zelenyi, L. M., and A. V. Milovanov (2004), Fractal topology and strange kinetics: From percolation theory to problems in cosmic electrodynamics, Phys. Usp., 47, 749-788.

Zelenyi, L., A. Artemyev, H. Malova, A. V. Milovanov, and G. Zimbardo (2008), Particle transport and acceleration in a time-varying electromagnetic field with a multi-scale structure, Phys. Lett. A, 372, 6284-6287.

Zimbardo, G., et al. (2010), Magnetic turbulence in the geospace environment, Space Sci. Rev., 156, 89-134.

A. V. Artemyev, A. A. Petrukovich, and L. M. Zelenyi, Space Research Institute, Russian Academy of Sciences, Profsoyusnaya St. 84/32, Moscow 117810, Russia. (ante0226@gmail.com; apetruko@iki.rssi.ru; lzelenyi@ iki.rssi.ru)

S. D. Rybalko, FEMTO/Optics Department, UMR 6174, CNRS, Universite de Franche-Comte, 16, route de Gray, F-25030 Besancon CEDEX, France. (rybalko@polly.phys.msu.ru)

G. Zimbardo, Dipartimento di Fisica, Universita della Calabria, I-87036 Arcavacatadi Rende, Italy. (gaetano.zimbardo@fis.unical.it) 